\title{
Analysis of the loss of vehicle motion stability in the conditions of steering control disturbance
}

\author{
Marian Dudziak ${ }^{1}$, Andrzej Lewandowski ${ }^{2}$, Michat Śledziński ${ }^{3, *}$ \\ ${ }^{1}$ State Vocational College in Kalisz, Poland \\ ${ }^{2}$ Institute of Forensic Research in Kraków, Poland \\ ${ }^{3}$ Poznań University of Technology, Poland
}

\begin{abstract}
Disturbances in the vehicle motion may be caused by different factors and in many cases are the reason for dangerous traffic incidents. Disturbances within the human-vehicle system are particularly hazardous. An innovative method was designed for analyzing and simulating the process of loss of vehicle motion stability after interference in the steering system, e.g. by acting on the steering wheel by the passenger. The subject of the study is the theoretical and experimental analysis of the vehicle motion path kinematics together with the duration of the disturbance, driver's reaction time and steering wheel turning angles. PC-Crash simulation software was employed for the purpose of studying the disturbance characteristics and their influence on the loss of vehicle motion stability. It is recognized that the studied issues are as yet poorly understood, the presented results expand our knowledge base in this area and can be employed for the purpose of analysis of actual traffic accidents.
\end{abstract}

Keywords: steering disturbance, steering wheel turning, loss of motion stability

\section{Introduction}

Loss of vehicle motion stability caused by disturbance of steering control is a known occurrence in traffic situations. This may be a result of acting on the steering wheel by the driver or the passenger. The practical analysis of traffic incidents recognizes cases in which the loss of vehicle motion stability occurred when the driver made a sudden turn of the steering wheel in order to react to a traffic situation, but may also be caused by momentary distraction (talking on the mobile phone, adjusting the audio system, conversation with the passenger). Accidental and interference with vehicle steering by the passenger which is unexpected by the driver are particularly dangerous. The usual result of such disturbance is the alteration of the vehicle's current trajectory. An attempt to counteract the disturbance by a sudden reaction of the driver is rarely effective. Such situation usually leads to loss of vehicle's motion stability and uncontrolled skidding off the road.

\footnotetext{
* Corresponding author: michal.sledzinski@put.poznan.pl

Reviewers: Czestaw Kundera, Mariana Pajtášová
} 
The dynamics of the vehicle's curvilinear motion caused by the disturbance, considering its stable and unstable areas, requires utilizing simulation software to analyze due to the degree of complexity [1-3]. The subject of interest to the authors was a detailed analysis of the vehicle motion with loss of motion stability caused by disturbance to the steering system - which occurs in actual traffic situations. The subject matter of this study was a theoretical and experimental analysis of vehicle trajectory kinematics together with the duration of the disturbance, driver's reaction time $t$ and steering wheel turning angles $\varphi$. The subject of examination was the speed range at which the driver may regain control of the vehicle following a rapid reaction to the disturbance caused.

\section{Assumptions, methodology and range of tests}

An innovative method was designed for analyzing and simulating the process of loss of vehicle motion stability after interference in the steering system, e.g. by acting on the steering wheel by the passenger. In establishing the methodology of study of the phenomenon of loss of vehicle's directional motion stability caused by disturbances to the steering process, road experiments were planned in order to establish the driver's motor characteristics during reaction to a sudden disturbance. These characteristics were described with the following parameters: driver's reaction time $t$ and turning angle of the steering wheel $\varphi$. The process of destabilizing the motion of the vehicle initialized by e.g. the passenger's interference is characterized by a complex dynamics of changing motion parameters. Therefore, it is planned to utilize PC-Crash - an advanced tool for software assisted graphical and computational analyses [4].

The software allows to carry out advanced simulations of vehicle motion dynamics. It utilizes a 3D vehicle model with 6 or 10 degrees of freedom. It is also possible to operate the software in two-dimensional mode with 3 degrees of freedom, which are: longitudinal and transverse displacement and yaw angle. The physical model of the wheel suspension system assumes zero-mass wheels suspended independently via flexible and shock absorbing components, placed parallel, reduced to the wheel center. It is also possible to introduce zeromass wheels loaded with moment of inertia relative to the wheel axis of rotation. The software model of the wheel suspension accounted only for the possibility of springing motion of the wheel parallel to the vehicle's vertical axis. On the other hand, wheel yaw occurs around the vertical axis passing through the center of the wheels and parallel to the vehicle's vertical axis. The software accounts for yaw kinematics according to Ackermann's system of dependencies. Additional weights acting on the vehicle are rigidly bound to the body. The vehicle force loads are described with generalized external forces, including:

- resultant of mass inertia force,

- grade resistance,

- resultant of aerodynamic force,

- forces of roadway reactions to wheels (vertical, longitudinal and transverse)

as well as moments of these forces in relation to the origin (vehicle center of mass). The vehicle weight was distributed into normal direction as well as tangent to the plane of movement of the vehicle. This allows to account for the longitudinal and transverse components of grade resistance. It is also possible to account for components of the aerodynamic force, wind direction and speed as well as intervals of air blast and silence [3]. Forces of roadway reaction to wheels are determined during the course of the simulation in accordance with the current condition of movement, accounting for local roadway traction and slope. The usual method of putting the vehicle to motion is by setting the required acceleration. Acceleration may be simulated by using engine characteristics and drive system ratios. Braking may be simulated using the declared characteristics for brake force distribution, as well as individually defined resistance parameters for each wheel. This allows 
to simulate damaged operation of the braking system or to introduce parameters resultant from the operation of the ABS system.

Modeling the interaction between the drive wheels (rubberized) and the roadway, in the conditions of determined and undetermined motion of the vehicle utilizes a linear or nonlinear model (TMeasy) [3]. It is possible to set the braking force of the drive wheels (wheels turning or locked) as well as to modify the characteristics of forces of roadway reaction to tire by changing the maximum tire drift angle. The operation of the ABS system is simulated in the software according to a universal algorithm which allows to meet the general criteria provided for this system. This method is sufficient to analyze the most typical cases of vehicle braking action during a traffic incident.

Proper utilization of the described software requires entering, apart from standard vehicle and roadway data, the motion characteristics for the driver's reaction (driver's reaction time $\mathrm{t}$ and steering wheel turning angle $\varphi$ ).

\section{Experimental studies}

\subsection{Stationary experiment configuration}

The study focused on the disturbance process in the vehicle's steering system through the steering wheel, the vehicle (OPEL ASTRA) was stationary - in "parked" position. The vehicle engine was kept running so that the power steering system is functioning. Persons participating in the experiment were the driver and the passenger occupying the front seat. Fig. 1 shows the diagram of steering wheel turning angles in the steering system of the vehicle.

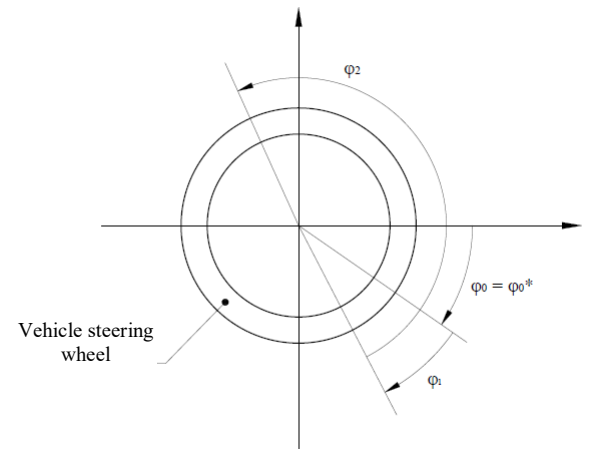

Fig.1. Diagram - steering wheel turning angles in the vehicle steering system, the angles $\varphi$ stand for: $\varphi_{0}$ - initial steering wheel turning angle (to the right) - corresponding to driving around a curve with steering wheel turn angle $\varphi_{0}{ }^{*}, \varphi_{1}$ - additional steering wheel turning angle (to the right) - disturbance introduced by the passenger, $\varphi_{2}-$ steering wheel turning angle (to the left) - driver's defensive reaction to counteract the disturbance introduced by the passenger

At the beginning of the experiment, the simulated driver at the initial time $t_{0}$ keeps the steering wheel turned to the right at the assumed angle $\varphi_{0}=\varphi_{0}{ }^{*}$. This angle corresponded to the actual steering wheel turn angle $\varphi_{0}{ }^{*}$ for the roadway curve. The turning of the steering wheel was filmed with an EXILIM camera by CASIO at 600 frames/s. Fig. 2a shows the initial stage of the disturbance process in the moment when the simulated passenger sitting on the right side of the driver begins to act on the right arm of the driver with his hand. This action caused a turn of the steering wheel by an additional angle of $\varphi_{1}$, as shown on Fig. $2 b$. 


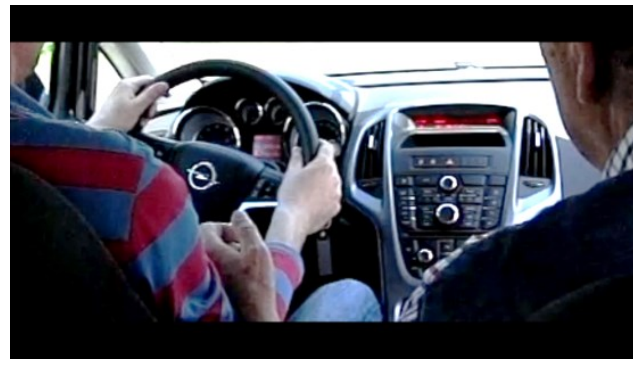

Fig. 2a. Start of the disturbance; the passenger on the right seat presses on the driver's right arm

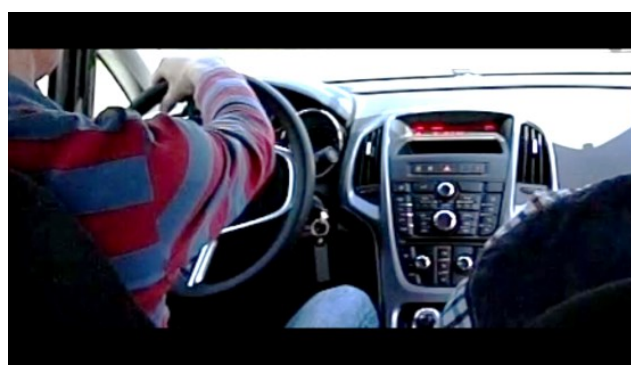

Fig. 2c. Maximum turn of the wheel to counteract the disturbance of the steering

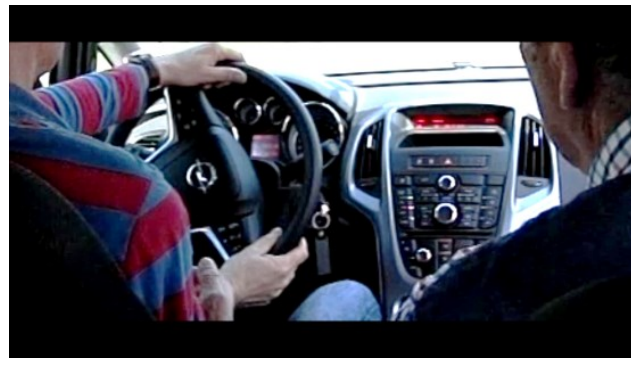

Fig. 2b. The disturbance action on the simulated driver's arm holding the steering wheel ceases

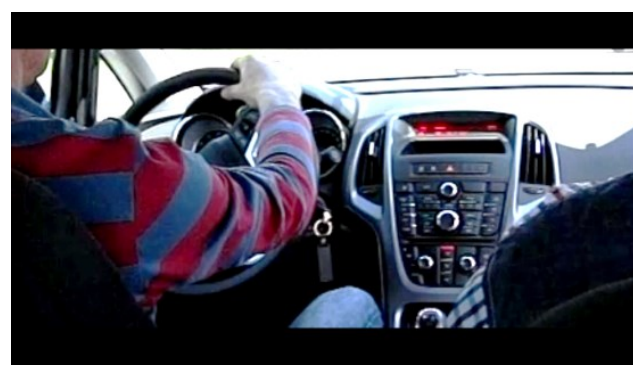

Fig. 2d. End position of the driver's reaction to the disturbance

The simulated driver was to carry out a defensive reaction to counteract the disturbance by quickly turning the steering wheel to the opposite direction - by angle $\varphi_{2}$ (Fig.1). Fig. 2c. shows the moment in which the steering wheel is maximally turned to the left, whereas Fig. $2 \mathrm{~d}$. shows the final turn of the steering wheel.

Based on the recorded material from the experiment, Movie Maker software was used to determine the time of individual motions of the steering wheel. The turning angles for positions shown on Figs. $(2 \mathrm{a}-2 \mathrm{~d})$ were established graphically. The aggregated results of the experiment are provided below in table 1 .

Table 1. Measurement results of the parameters of driver's reaction to disturbance in the steering system under stationary conditions

\begin{tabular}{|c|c|c|c|}
\hline & duration t & $\begin{array}{c}\text { steering wheel } \\
\text { turn angle } \boldsymbol{\varphi}\end{array}$ & $\begin{array}{c}\text { steering wheel } \\
\text { turn speed } \mathbf{~}_{\mathbf{k}}\end{array}$ \\
\hline & {$[\mathrm{s}]$} & degrees & degrees/s \\
\hline disturbance & 0,29 & 30 (to the right) & 103 \\
\hline reaction & 0,46 & 120 (to the left) & 261 \\
\hline
\end{tabular}

The driver's motion characteristics as indicated above were utilized in the simulation study.

\subsection{Dynamic experiment configuration}

The aim of the subsequent experimentation was to determine the maximum parameters of steering wheel turning angle, within the range of stable vehicle motion [5-6]. The experiment tested vehicle behavior at set speeds of $40 \mathrm{~km} / \mathrm{h}$ and $50 \mathrm{~km} / \mathrm{h}$. Next, the vehicle was set to motion at a curvilinear trajectory through rapid, alternating turns of the steering wheel to the left and to the right. The study was carried out by utilizing the EXILIM camera to register the steering wheel turning angles.

The results of the carried-out tests are provided in Table 2. 
Table 2. Measurement results of the driver's motion parameters during the vehicle's yaw motion

\begin{tabular}{|c|c|c|c|}
\hline vehicle speed v & $\begin{array}{c}\text { steering wheel } \\
\text { turn angle } \boldsymbol{\varphi}\end{array}$ & $\begin{array}{c}\text { steering wheel } \\
\text { turn time t }\end{array}$ & $\begin{array}{c}\text { steering wheel } \\
\text { turn speed vk }\end{array}$ \\
\hline $\mathrm{km} / \mathrm{h}$ & degrees & $\mathrm{s}$ & degrees/s \\
\hline 40 & 88 & 0,39 & 225 \\
\hline 40 & 90 & 0,42 & 215 \\
\hline 50 & 137 & 0,46 & 297 \\
\hline 50 & 121 & 0,40 & 304 \\
\hline
\end{tabular}

The results indicate the possible range of driver's reaction in traffic conditions (for the assumed vehicle speeds), by executing a turning motion with the steering wheel. The angular range is $90-120$ degrees. Whereas the duration of the maneuver fits in the narrow range of 0,39-0,46 s. This corresponds to steering wheel turning speed 215-304 degrees/s. Identifying these parameters is necessary to carry out an analysis of vehicle motion using the simulation software.

\section{Simulation studies}

The aim of the simulation studies was to carry out an analysis of vehicle motion stability under conditions of disturbance acting on the steering wheel system.

A disturbance was programmed within the simulation, assuming an action by the passenger to disturb the process of steering the vehicle by the driver, within the range determined during stationary experiment configuration. Driver's reaction was accounted for, which typically entails a rapid turning of the steering wheel in the direction opposite to the disturbance. Simulation studies were carried out using PC-Crash software. It simulated the driver's attempt to maintain the initial vehicle trajectory. The driver's reaction process observed during experimentation was divided into stages by providing the following input data:

- vehicle speed $40 \mathrm{~km} / \mathrm{h}$,

- disturbance action parameters: duration $0.3 \mathrm{~s}$; steering wheel turn angle -30 degrees (to the right),

- driver's reaction parameters (stage I): duration $0.46 \mathrm{~s}$; steering wheel turn angle 110 degrees (to the left),

- driver's reaction parameters (stage II): steering wheel turn duration $0.86 \mathrm{~s}$, steering wheel turn angle -110 degrees (to the right).

Simulation results for speed $40 \mathrm{~km} / \mathrm{h}$ are presented graphically on Figs. 3 and 4.

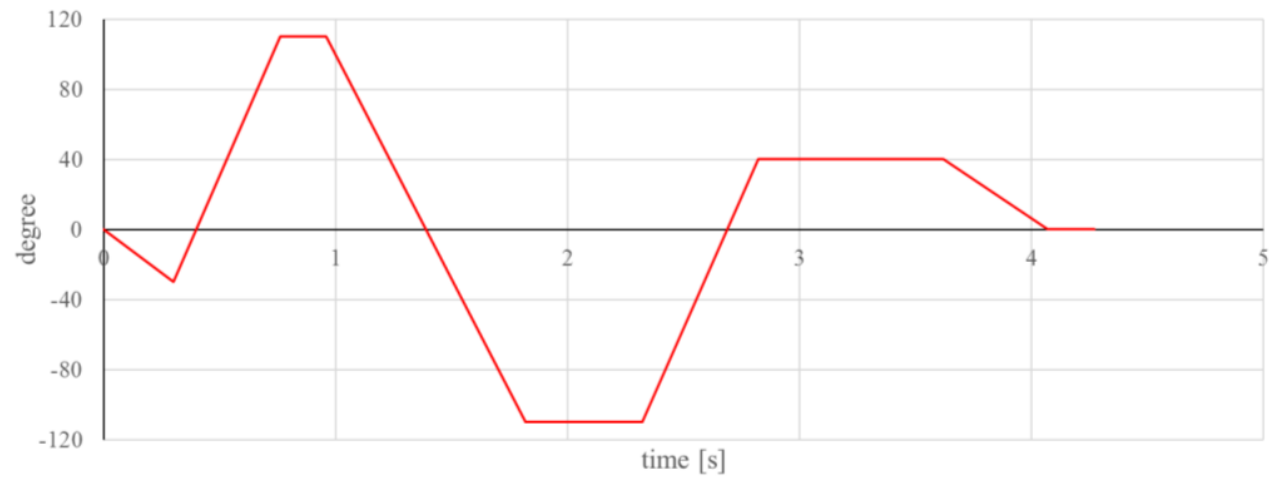

Fig.3. Graph illustrating steering wheel turn parameter change 
The graph shows the simulation input data in graphical form together with the assumed driver's reaction times between the stages. See below for a graphical representation of the vehicle's trajectory caused by the disturbance and the driver's action.

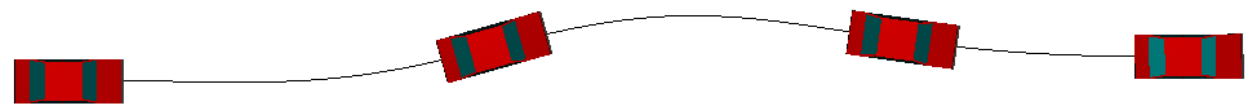

Fig. 4. Simulated vehicle motion trajectory at $40 \mathrm{~km} / \mathrm{h}$

The analysis of the vehicle's motion presented on Fig. 4. indicates that there was no wheel skidding at $40 \mathrm{~km} / \mathrm{h}$.

The simulation of vehicle movement indicates that the changes in trajectory do not correspond to the momentary changes in the turn of the steering wheel. This is a consequence of a complex vehicle motion dynamics within the driver - vehicle - arrangement. In the course of the simulation, the subsequent stages of the driver's reaction were selected so that the vehicle returns to the initial trajectory at the speed $40 \mathrm{~km} / \mathrm{h}$.

With the same initial data, the simulation was carried out for vehicle speed $50 \mathrm{~km} / \mathrm{h}$, as shown on Fig. 5. In this scenario, a short-term skidding of the wheels was observed, as seen on the image, however it did not have a substantial effect on the vehicle resuming to drive forward. This indicates limit values of vehicle wheel lateral acceleration for the given traction.

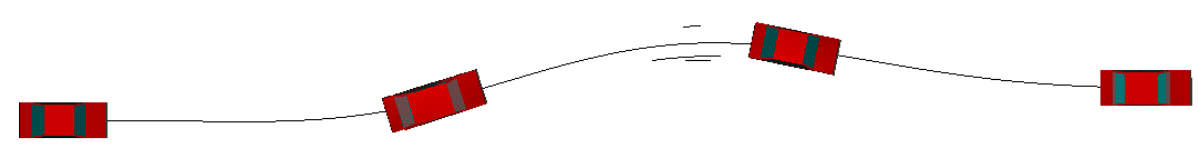

Fig. 5. Changes in vehicle trajectory at $50 \mathrm{~km} / \mathrm{h}$

After performing numerous simulations for other vehicle speed values, it was determined that range of vehicle skidding increases together with the movement speed.

Fig. 6. shows an example simulation performed for the speed $90 \mathrm{~km} / \mathrm{h}$.

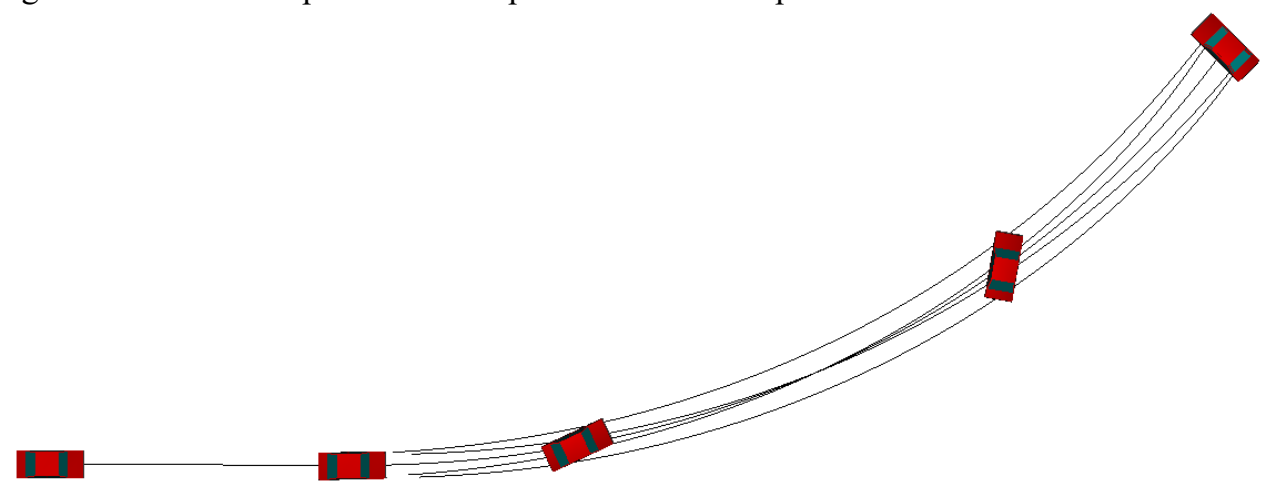

Fig. 6. Changes in vehicle trajectory at $90 \mathrm{~km} / \mathrm{h}$

The performed simulation demonstrates that the vehicle loses directional stability during the driver's initial reaction stage to the disturbance and it is not possible to restore the stability of its motion.

It is therefore demonstrated that for the set parameters of driver's reaction to the disturbance parameters there exists a limit value of vehicle movement speed at which it is still possible to restore the initial movement trajectory. 


\section{Conclusion}

The performed analysis demonstrated the usefulness of static and dynamic experimental studies for the purpose of determining initial parameters for disturbance and driver's reaction. The advantages of employing PC-Crash simulation software for analyzing vehicle motion dynamics and the loss of stability due to disturbance to the steering system by the passenger was confirmed. It was demonstrated that if the limit values of parameters characterizing the vehicle motion such as: driving speed, lateral acceleration, traction conditions as well as range and time of the driver's maneuvers to counteract the disturbance are exceeded, there is no possibility for the vehicle to return to stable motion, regardless of the driver's action on the steering wheel. The presented results expand our knowledge base in this area and can be employed for the purpose of reconstruction of actual traffic accidents in which such types of disturbances occur.

\section{References}

1. G. Beauchamp, D. Hessel, N. A. Rose, S. J. Fenton, Determining Vehicle Steering and Braking from Yaw Mark Stations. SAE 2009-01-0092,

2. G. Amirault, S. Maclnnis, Variability of Yaw Calculations from Field Testing. SAE 2009-01-0103

3. S. Mary, R. Douglas, A. Dennis, A Comparison Study of Skid and Yawmarks. SAE 890635

4. W. Wach, Symulacja wypadków drogowych $w$ programie PC-Crash. IES, Kraków (2009),

5. A. Lewandowski, Limit velocity during car skidding process. Technolog 4, ročnik 5 (2013)

6. Praca zbiorowa, Wypadki drogowe - Vademecum biegłego sądowego. IES Kraków, (2011) 